\title{
Viral counterattack against the host innate immune system
}

Cell Research (2013) 23:735-736. doi:10.1038/cr.2013.32; published online 12 March 2013

\begin{abstract}
Paramyxoviruses evade antiviral immune response using a small nonstructural protein, $\mathrm{V}$, which binds to the host dsRNA sensor MDA5 and prevents it from activating the interferon signaling pathway. A recent crystal structure of the $\mathrm{V}$ protein in complex with MDA5, published in Science, revealed that $\mathrm{V}$ disrupts the structure of MDA5 and is integrated into the MDA5 protein fold, providing an intriguing new example of viral mimicry as a countermeasure against the host immune system.
\end{abstract}

Effective immune response against viruses requires efficient recognition of viral RNAs by host innate immune receptors. To defend against such recognition, viruses encode proteins that either sequester viral RNAs from the host immune system, or directly bind and inhibit (or cleave) immune receptors or other molecules involved in the antiviral signaling pathways. Such viral proteins include VP35 from Ebola virus and NS1 from influenza A virus, which bind to viral double-stranded RNA (dsRNA); K3L from vaccinia virus, which binds to a viral RNA receptors, PKR, and NS3-4A from hepatitis $\mathrm{C}$ virus, which cleaves MAVS, a common signaling adaptor for viral RNA receptors, RIG-I and MDA5. Paramyxoviruses encode a nonstructural protein, $\mathrm{V}$, that binds to MDA5 and prevents viral RNA recognition and interferon signal activation. Understanding the complex arms race between the host and viruses would thus require detailed knowledge of both the receptor:viral RNA and receptor:viral inhibitor interactions.

MDA5 and RIG-I are close paralogs, and together represent a major family of viral RNA receptors in the cytoplasm. Despite the same domain architecture and signaling pathway, MDA5 and RIG-I perform non-redundant functions in antiviral immunity partly by recognizing distinct classes of viruses and viral RNAs. RIG-I recognizes short dsRNA with the $5^{\prime}$ triphosphate group and blunt ends, which are often present in genomic RNAs of a wide range of viruses [1]. In contrast, MDA5 recognizes long duplex RNA in the genome of dsRNA viruses or replicative form of picornaviruses [2]. While paramyxoviruses were initially thought to be recognized primarily by RIG-I, and not MDA5, studies with MDA5 knock-out mice showed the importance of MDA5 in antiviral response against these viruses [3].

How does MDA5 recognize viral dsRNA? We found that MDA5 assembles into linear filaments along the length of dsRNA and that this filament formation is required for antiviral signal activation as it promotes oligomerization of the signaling domain, caspase activation recruitment domain (CARD) [4, 5]. Furthermore, filament formation allows MDA5 to regulate its interaction with dsRNA according to the length of dsRNA as it disassembles from filament termini during ATP hydrolysis [6]. Our crystal structure of MDA5 bound to dsRNA further revealed that MDA5 forms an open ring structure [4] (Figure 1), which is distinct from the structure of RIG-I that caps the dsRNA end.

The recent crystal structure of MDA5 in complex with V from parainfluenza virus 5 reported by Motz et al. [7] now provides additional insights into how the virus prevents MDA5 from recognizing viral RNAs and activating antiviral signaling pathways. While previous studies showed that $\mathrm{V}$ prevents MDA5 from binding to dsRNA, whether $\mathrm{V}$ simply blocks the RNA binding site or employs other more intricate mechanisms was unclear. The structure of the MDA5:V complex showed that $\mathrm{V}$ contains the $\beta$-hairpin structure at the $\mathrm{C}$-terminus rigidly held by two zinc ions, and this $\beta$-hairpin of $\mathrm{V}$ mimics and replaces a portion of the beta-sheet structure in MDA5 (Figure 1), revealing an unusual viral mimicry mechanism. The conformation of MDA5 in the MDA5:V structure is incompatible with dsRNA binding, filament formation and ATP hydrolysis, therefore inhibiting the antiviral function of MDA5. Furthermore, the authors identified a single amino acid, R803, in MDA5 to be necessary and sufficient to interact with $\mathrm{V}$, a finding independently confirmed by the recent biochemical studies [8]. R803 is also present in LGP2, another paralogous receptor inhibited by $\mathrm{V}$, but not in RIG-I, and placement of Arg at the equivalent site in RIG-I allowed V to inhibit RIG-I along with MDA5 and LGP2. It would be interesting to examine whether the lack of the Arg residue in RIG-I reflects evolutionary pressure to evade viral mimicry. In fact, such positive selection has been proposed 


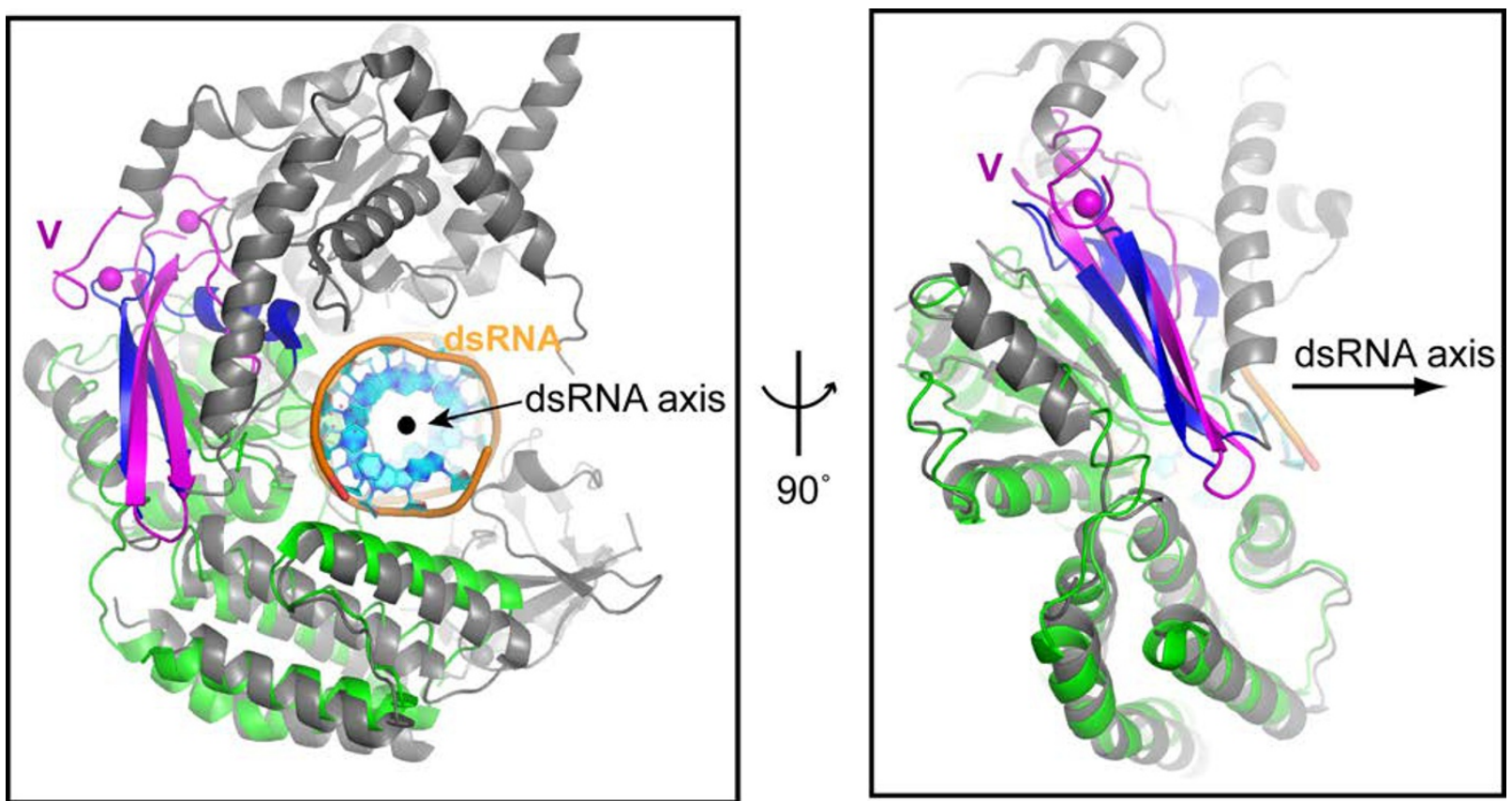

Figure 1 Superposition of the structures of MDA5:V [7] and MDA5:dsRNA [4] complexes. While the overall structure of MDA5 (green) bound to the $\mathrm{V}$ protein (magenta) aligns well with that of MDA5 (grey and blue) bound to dsRNA (orange backbone and cyan base), the C-terminal domain of $\mathrm{V}$ displaces two $\beta$ strands (blue) in MDA5 in the MDA5:dsRNA complex structure. This displacement disrupts the motif $\mathrm{VI}$ in the helicase domain critical for ATP hydrolysis and prevents MDA5 from wrapping around dsRNA, forming filaments and ultimately activating the interferon signal pathway. Two magenta spheres represent zinc ions coordinated at the C-terminal domain of $\mathrm{V}$.

to contribute to the rapid evolution of PKR [9].

Inhibition of MDA5 and LGP2 is not the only function of the $\mathrm{V}$ proteins; $\mathrm{V}$ has been shown to bind to cellular protein DDB1, a component of a ubiquitin E3 ligase, which then facilitates ubiquitination and degradation of STAT proteins involved in antiviral signaling pathways [10]. Such functional versatility of $\mathrm{V}$ is reminiscent of that observed with other viral proteins, such as NS1 from influenza A, which binds to viral dsRNA and a number of host factors to evade antiviral immune response and perturb cellular and viral mRNA processing. The functional flexibility of these viral proteins likely reflects the evolutionary pressure of viruses to maintain their small genome size while defending against the variety of host factors, and highlights diversity and ingenuity of viral survival and persistence mechanism, as exemplified by the structure of the MDA5:V complex.

\section{Bin $\mathrm{Wu}^{1,2}$, Sun Hur ${ }^{1,2}$}

${ }^{1}$ Department of Biological Chemistry and Molecular Pharmacology, Harvard Medical School, Boston, MA 02115, USA; ${ }^{2}$ Program in Cellular and Molecular Medicine, Boston Children's Hospital, Boston, MA 02115, USA

Correspondence: Sun Hur

E-mail: hur@idi.harvard.edu

\section{References}

1 Schlee M, Roth A, Hornung V, et al. Immunity 2009; 31:25-34.

2 Kato H, Takeuchi O, Mikamo-Satoh E, et al. $J$ Exp Med 2008; 205:1601-1610.

3 Gitlin L, Benoit L, Song C, et al. PLoS Pathog 2010; 6:e1000734.

4 Wu B, Peisley A, Richards C, et al. Cell 2013; 152:276-289.

5 Peisley A, Lin C, Wu B, et al. Proc Natl Acad Sci USA 2011; 108:21010-21015.

6 Peisley A, Jo MH, Lin C, et al. Proc Natl Acad Sci USA 2012; 109:E3340-E3349.

7 Motz C, Schuhmann KM, Kirchhofer A, et al. Science 2013; 339:690-693.

8 Rodriguez KR, Horvath CM. J Virol 2013; 87:2974-2978.

9 Elde NC, Child SJ, Geballe AP, et al. Nature 2009; 457:485-489.

10 Li T, Chen Z, Garbutt KC, et al. Cell 2005; 124:105-117. 